\section{Reversible Oxidation of Copper(I) lodide in the Presence of Imidazole}

THE importance of copper ions in natural redox systems is well known. For example, the oxidation of diphenols by molecular oxygen occurs by way of a $\mathrm{Cu}$ (I) to $\mathrm{Cu}$ (II) oxidation in the diphenol oxidase. We report here a simple $\mathrm{Cu}(\mathrm{I})-\mathrm{Cu}(\mathrm{II})$ redox system, with atmospheric oxygen as the oxidizing agent, which is readily reversible in biologically attainable conditions. This reversibility arises because of the presence of two different types of ligand, imidazole, which strongly favours the higher oxidation state, and iodide, which normally reduces copper(II), yielding insoluble cuprous iodide.

Exposure to air of a suspension of copper $(\mathbf{I})$ iodide in a concentrated solution of imidazole in ethanol results in the formation of a blue, paramagnetic solution, indicating the formation of copper(II) ions. The stability of the solution towards reduction is decreased by a reduced imidazole to metal ratio, by increasing temperature, and by decreasing the polarity of the surrounding medium, small changes in these factors producing a drastic change in the position of the redox equilibrium.

Iodine may also be used as the oxidizing agent in ethanolic solution, provided an excess of imidazole is present. In this case $\mathrm{Cu}$ (imidazole $)_{4} \mathrm{I}_{2}$. imidazole ${ }_{2}$ has been isolated as blue crystals from the oxidized solution. Both the solution and the solid have electronic spectra typical of copper(II) ions in tetragonal environments.

With $\mathrm{N}$-methylimidazole as ligand, a very similar redox system occurs, but the solid isolated has the stoichiometry $\mathrm{Cu}(\mathrm{N} \text {-methylimidazole })_{4} \mathrm{I}_{2}$. Benzimidazole and 2-methylimidazole, however, do not stabilize the copper(II) species in the presence of iodide ions. It seems likely that in these cases a longer, and weaker, copper-nitrogen bond would be necessary because of the size of the ligand.

Table 1. ELECTRONIC SPECTRA AND EPR RESCLTS

\begin{tabular}{|c|c|c|c|c|c|}
\hline & $\begin{array}{c}\text { Electronic } \\
\text { spectrum } \\
\left(\mathrm{cm}^{-1} \times 10^{3}\right)\end{array}$ & $g_{11}$ & $g_{\perp}$ & $\begin{array}{l}A_{11} \\
\left(10^{-4}\right. \\
\left.\mathrm{cm}^{-1}\right)\end{array}$ & $\begin{array}{l}A_{1} \\
\left(10^{-4}\right. \\
\left.\mathrm{cm}^{-1}\right)\end{array}$ \\
\hline $\mathrm{Cu}$ (imidazole $)_{4} \mathrm{I}_{2}$ & $18 \cdot 2, \sim 14.5 \mathrm{sh}$ & \multicolumn{2}{|c|}{$g \mathrm{v}=2 \cdot 07^{*}$} & & \\
\hline Cu(imidazole $)_{4} \mathrm{I}_{2}$. imida ile $_{2}$ & $16 \cdot 8 \mathrm{br}$ & \multicolumn{2}{|c|}{$g_{1 \mathrm{v}}=2.06^{*}$} & & \\
\hline , $\quad\left(\right.$ in $\left.\mathrm{H}_{2} \mathrm{O}\right) \dagger$ & $17 \cdot 0$ & $2 \cdot 26$ & $2 \cdot 09$ & 174 & 20 \\
\hline ,, (in ethanol) & $15 \cdot 8$ & $2 \cdot 27$ & $2 \cdot 07$ & 179 & 17 \\
\hline ," (in solid Co(imidazole $\left.)_{6} \mathrm{I}_{2}\right)$ & - & $2 \cdot 25$ & $2 \cdot 02$ & 173 & - \\
\hline $\mathrm{Cu}(\mathrm{N} \text {-methylimidazole })_{4} \mathrm{I}_{2}$ & $16 \cdot 7, \sim 14 \cdot 3 \mathrm{sh}$ & $2 \cdot 27$ & $2 \cdot 00$ & 164 & - \\
\hline,$\quad\left(\right.$ in $\left.\mathrm{H}_{2} \mathrm{O}\right) \ddagger$ & $16 \cdot 3$ & $2 \cdot 27$ & $2 \cdot 07$ & 165 & 30 \\
\hline, (in ethanol) $\ddagger$ & $15 \cdot 5$ & $2 \cdot 27$ & $2 \cdot 06$ & 170 & $>10$ \\
\hline
\end{tabular}

The EPR spectra of these systems (see Table 1) are generally similar to that of $\mathrm{Cu}$ (imidazole $)_{4}\left(\mathrm{NO}_{3}\right)_{2}$ in solution $^{1}$, but nitrogen--hyperfine structure is observed in some media. (We have used the method of Kneubühl ${ }^{2}$ to estimate the value of $g_{\perp}$ for the solids, which leads to slightly lower values than those of ref. 1.) For aqueous solutions of $\mathrm{Cu}(\mathrm{N} \text {-methylimidazole })_{4} \mathrm{I}_{2}$ containing a small excess of ligand, the nitrogen hyperfine structure occurs weakly, with separations of about $11 \mathrm{G}$, on the $m_{1}=-\frac{3}{2}$ component of $g_{0}$, as found ${ }^{3}$ for $\mathrm{Cu}$ (pyridine $)_{4}{ }^{2+}$. In the absence of excess ligand, the copper hyperfine components are ill defined, but the nitrogen hyperfine structure on the high-field line is better resolved. The ethanol solutions of both the imidazole and the $\mathrm{N}$-methylimidazole complexes show nitrogen hyperfine structure at $-160^{\circ}$, but the resolution and intensity pattern are sensitive to the quality of the glass formed. On $g_{\perp}$ up to fifteen lines were observed, with separations of about $15 \mathrm{G}$. For the best glasses of the imidazole complex, five weak but reproducible peaks of $7 \mathrm{G}$ separation are observed on the lowestfield copper hyperfine component of $g_{11}$.
We thank the Agricultural Research Council for financial support.

\section{M. L. Goodgame* \\ M. Goodgame \\ G. W. Rayner Canham}

Inorganic Chemistry Research Laboratories, Imperial College, London SW7.

Received February 24; revised Mareh 26, 1969.

* Present address: Department of Inorganic Chemistry, Cniversity of Melbourne, Parkville 3052, Victoria, Australia.

1 Malmström, B. G., and Vănngård, T., J. Mol. Biol., 2, 118 (1960).

${ }^{2}$ Kneubühl, F. K., J. Chem. Phys., 33, 1074 (1960).

${ }^{3}$ Wuthrich, K., Helv. Chim. Acta, 49, 1400 (1966).

\section{Silicon in Iron Meteorites and the Earth's Core}

WE have searched for silicon in the metal phase of several iron meteorites in an attempt to test those Earth models which assume that silicon occurs as a major component in the core of the Earth ${ }^{1,2}$. The experimental technique was activation analysis, based on the fast neutron reaction ${ }^{28} \mathrm{Si}(n, p)^{28} \mathrm{Al}$. This reaction is known to $\mathrm{be}^{3}$ an important contaminant in the determination by the slow-neutron induced reaction ${ }^{27} \mathrm{Al}(n, \gamma){ }^{28} \mathrm{Al}$ of $\mathrm{Al}$ in stone meteorites, in which the silicon content is well known and in which the observed ${ }^{28} \mathrm{Al}$ activity can be corrected for the contribution from silicon. In the iron meteorites, any ${ }^{28} \mathrm{Al}$ activity would have to be attributed to an unknown mixture of silicon and aluminium. As it turns out, however, no activity is seen and so limits can be set to both the aluminium and silicon contents of the iron meteorites.

Approximately $\mathrm{l} \mathrm{g}$ samples of iron meteorites, together with aluminium and silicon standards, were irradiated $^{3}$ in the rabbit facility of the Cornell University reactor for one minute at a flux of about $10^{10}$ neutrons $\mathrm{cm}^{-2} \mathrm{~s}^{-1}$. The samples were then counted on a 256 channel analyser with an $\mathrm{NaI}$ detector focused on the $1.83 \mathrm{MeV}$ gamma ray of ${ }^{28} \mathrm{Al}$. In no case did the activity exceed background, although I calculate that it would have been easy to detect 20 counts above background, corresponding to about $5 \times 10^{-5} \mathrm{~g}$ silicon or about 50 p.p.m. for the $1 \mathrm{~g}$ samples. In the particular experimental configuration used, aluminium provides about five times the activity per gram so that limits of about 10 p.p.m. aluminiun can be set. The iron samples measured were octahedrites (Arispe, Canyon Diablo, Norfolk, Norfork), hexahedrites (Bennett County, Coahuila, El Burro, Sikhote Alin, Smithonia) and ataxites (Indian Valley, Santa Rosa).

Although it would be an oversimplification to consider the core of the Earth as completely comparable with iron meteorites, if there is a meteorite analogy of any kind, the iron meteorites should be considered before those of some other class. Ringwood ${ }^{4}$ and later workers ${ }^{5,6}$ found abundances of 1-4 weight per cent in the metal phase of enstatite chondrites, but no silicon in the metal phase of ordinary chondrites. Ringwood concluded that this was evidence of the possible existence of silicon in the core of the Earth. I consider that the limit set in this work, less than 50 p.p.m. silicon in the metal phase of iron meteorites, is more likely to be relevant to the Earth core problem. Such a limit argues against high ( $\sim 20$ per cent) silicon abundances in the core of the Earth required by the models of refs. 1 and 2.

The experimental work was done during 1963-64 while I was at Cornell University, and was partially supported by the US AEC. The meteorite samples were obtained from Ward's, Arizona State University, Chicago Natural 